University of Wollongong

Research Online

Australian Institute for Innovative Materials -

Papers

Australian Institute for Innovative Materials

$1-1-2014$

\title{
Achieving single domain relaxor-PT crystals by high temperature poling
}

Fei Li

Xi'an Jiaotong University

Linghang Wang

Xi'an Jiaotong University

Li Jin

Xi'an Jiaotong University

Zhuo Xu

Xi'an Jiaotong University

Shujun Zhang

Pennsylvania State University, shujun@uow.edu.au

Follow this and additional works at: https://ro.uow.edu.au/aiimpapers

Part of the Engineering Commons, and the Physical Sciences and Mathematics Commons

Research Online is the open access institutional repository for the University of Wollongong. For further information contact the UOW Library: research-pubs@uow.edu.au 


\title{
Achieving single domain relaxor-PT crystals by high temperature poling
}

\begin{abstract}
Single domain relaxor-PT crystals are important from both fundamental and application viewpoints. Compared to domain engineered relaxor-PT crystals, however, single domain crystals are prone to cracking during poling. In this paper, based on the analysis of the cracking phenomenon in [001] poled tetragonal 0.25Pb(In0.5Nb0.5)03-0.37Pb(Mg 1/3Nb2/3)03-0.38PbTiO3 (PIN-PMN-PT) crystals, the non- $180^{\circ}$ ferroelastic domain switching was thought to be the dominant factor for cracking during the poling process. A high temperature poling technique, by which the domain switching can be greatly avoided, was proposed to achieve the single domain relaxor-PT crystals. By this poling approach, a quasisingle domain crystal was obtained without cracks. In addition, compared to room temperature poling, the high temperature poled PIN-PMN-PT crystals showed improved electromechanical properties, i.e., a low dielectric loss, a low strain-electric field hysteresis and a high mechanical quality factor, demonstrating a beneficial poling approach. This journal is the Partner Organisations 2014.
\end{abstract}

\section{Keywords}

pt, relaxor, single, achieving, crystals, domain, high, temperature, poling

Disciplines

Engineering | Physical Sciences and Mathematics

\section{Publication Details}

Li, F., Wang, L., Jin, L., Xu, Z. \& Zhang, S. (2014). Achieving single domain relaxor-PT crystals by high temperature poling. CrystEngComm, 16 (14), 2892-2897. 


\section{CrystEngComm}

\section{RELAXOR-PT ORYSTAL \\ Tre}

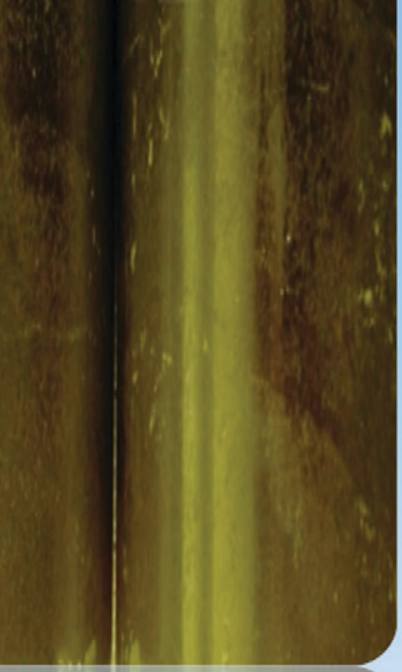

0

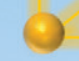

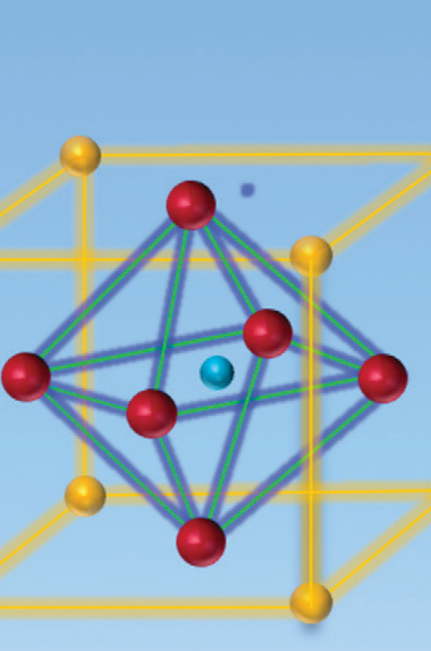

4

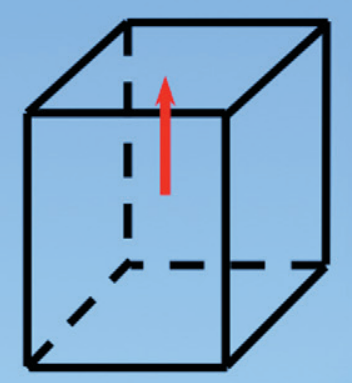

0
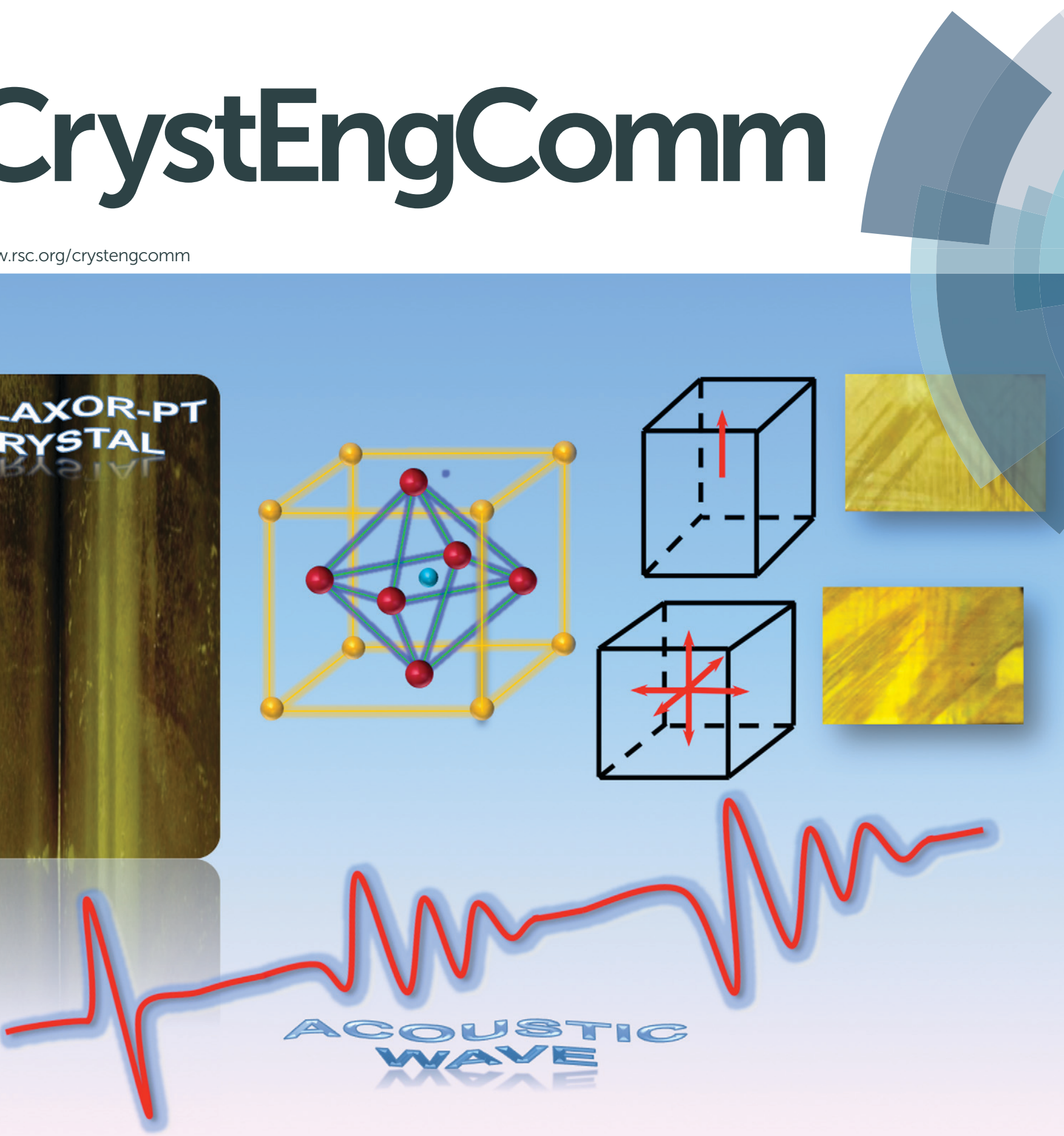


\section{CrystEngComm}

Cite this: CrystEngComm, 2014, 16, 2892

Received 16th November 2013, Accepted 27th January 2014

DOI: $10.1039 / c 3 c e 42330 a$

www.rsc.org/crystengcomm

\section{Achieving single domain relaxor-PT crystals by high temperature poling}

\author{
Fei Li, ${ }^{a}$ Linghang Wang, ${ }^{a}$ Li Jin, ${ }^{a}$ Zhuo $\mathrm{Xu}^{\mathrm{a}}$ and Shujun Zhang*b
}

Single domain relaxor-PT crystals are important from both fundamental and application viewpoints. Compared to domain engineered relaxor-PT crystals, however, single domain crystals are prone to cracking during poling. In this paper, based on the analysis of the cracking phenomenon in [001] poled tetragonal $0.25 \mathrm{~Pb}\left(\mathrm{In}_{0.5} \mathrm{Nb}_{0.5}\right) \mathrm{O}_{3}-0.37 \mathrm{~Pb}\left(\mathrm{Mg}_{1 / 3} \mathrm{Nb}_{2 / 3}\right) \mathrm{O}_{3}-0.38 \mathrm{PbTiO}_{3}$ (PIN-PMN-PT) crystals, the non-180 ferroelastic domain switching was thought to be the dominant factor for cracking during the poling process. A high temperature poling technique, by which the domain switching can be greatly avoided, was proposed to achieve the single domain relaxor-PT crystals. By this poling approach, a quasi-single domain crystal was obtained without cracks. In addition, compared to room temperature poling, the high temperature poled PIN-PMN-PT crystals showed improved electromechanical properties, i.e., a low dielectric loss, a low strain-electric field hysteresis and a high mechanical quality factor, demonstrating a beneficial poling approach.

\section{Introduction}

Relaxor-PbTiO ${ }_{3}$ (relaxor-PT) based ferroelectric single crystals, such as $\mathrm{Pb}\left(\mathrm{Mg}_{1 / 3} \mathrm{Nb}_{2 / 3}\right) \mathrm{O}_{3}-\mathrm{PbTiO}_{3}$ (PMN-PT) and $\mathrm{Pb}\left(\mathrm{In}_{1 / 2} \mathrm{Nb}_{1 / 2}\right) \mathrm{O}_{3}-\mathrm{Pb}\left(\mathrm{Mg}_{1 / 3} \mathrm{Nb}_{2 / 3}\right) \mathrm{O}_{3}-\mathrm{PbTiO}_{3}$ (PIN-PMN-PT), have attracted considerable interest because of their ultrahigh piezoelectric activities, far outperforming the state-of-the-art polycrystalline $\mathrm{Pb}(\mathrm{Zr}, \mathrm{Ti}) \mathrm{O}_{3}$ (PZT) ceramics. ${ }^{1-6}$ Over the last decade, extensive studies have been focused on the piezoelectric response of domain engineered crystals, especially for [001] poled rhombohedral relaxor-PT crystals, with longitudinal piezoelectric coefficient $d_{33}$ and electromechanical coupling factor $k_{33}$ being on the order of $>1500 \mathrm{pC} \mathrm{N}^{-1}$ and $>0.9$, respectively. Ferroelectric phase instability is thought to be responsible for the high piezoelectric activity in relaxor-PT crystals, ${ }^{7,8}$ which can be induced by the phase transition points (for example, morphotropic phase boundary and polymorphic phase transition) and relaxor component. ${ }^{2}$ In addition to the high piezoelectric response, a hysteresis-free strain-electric field behavior is another important characteristic for relaxor-PT crystals, which is inherently associated with the domain engineered configuration. In rhombohedral (R) crystals, there are eight degenerate domain variants, with the polar vectors (spontaneous polarizations) along one of the $<111>$ directions. As R crystals are poled

\footnotetext{
${ }^{a}$ Electronic Materials Research Laboratory, Key Laboratory of the Ministry of Education and International Center for Dielectric Research, Xi'an Jiaotong University, Xi'an 710049, China

${ }^{b}$ Materials Research Institute, Pennsylvania State University, University Park, Pennsylvania 16802, USA. E-mail: soz1@psu.edu
}

along the non-polar [001] direction, four of the eight domain variants are energetically favored by the poling field, with polar vectors along the [111], [īi11], [i111], and [1111] directions. This domain structure is labelled as " $4 \mathrm{R}$ ". ${ }^{9}$ Because these four domain variants are equivalent to the poling [001] direction, the domain structure is stable as the electric field is applied along the [001] direction, resulting in a hysteresis-free strainelectric field response. ${ }^{1}$ Similarly, [001] poled orthorhombic and [111] poled tetragonal relaxor-PT crystals possess " $4 \mathrm{O}$ " and "3T" domain engineered configurations, respectively, ${ }^{9}$ which exhibit hysteresis-free strain characteristics. Thus, domain engineered relaxor-PT crystals greatly benefit the piezoelectric transducer and actuator applications. ${ }^{10-12}$

Compared to the domain engineered crystals, single domain relaxor-PT crystals, though not receiving much attention, are important in both scientific and application aspects. In the scientific aspect, the data of the single domain crystals are essential for studying domain engineered crystals and polycrystalline ceramics. Based on the single domain data, the intrinsic piezoelectric response of crystals (regardless of the domain wall motion) and the anisotropy of the crystal properties can be derived. ${ }^{2,13,14}$ From the application viewpoint, on the other hand, single domain crystals exhibit many advantages, such as high thickness shear piezoelectric and pyroelectric properties. ${ }^{15-21}$ As given in Table $1,{ }^{2,15}$ the shear piezoelectric activities of single domain crystals are much higher than those of domain engineered crystals, with $d_{15}$ and $k_{15}$ being on the order of $\sim 2000 \mathrm{pC} \mathrm{N}^{-1}$ and $>90 \%$, respectively. In addition, compared with the longitudinal piezoelectric response of domain engineered crystals, the shear 
Table 1 Comparison of shear piezoelectricity and pyroelectric response among domain engineered crystals ([001] poled PMN-0.30PT crystals) and single domain crystals. Coefficients $\varepsilon_{11}^{\top} / \varepsilon_{22}^{T}, s_{55}^{E} / s_{44}^{E}, d_{15} / d_{24}$, and $p$ are dielectric, elastic, piezoelectric and pyroelectric coefficients, respectively

\begin{tabular}{|c|c|c|c|c|c|c|c|}
\hline Materials & $\varepsilon_{11}^{\mathrm{T}} / \varepsilon_{0}$ & $s_{55}^{\mathrm{E}}\left(\mathrm{pm}^{2} \mathrm{~N}^{-1}\right)$ & $d_{15}\left(\mathrm{pC} \mathrm{N}^{-1}\right)$ & $\varepsilon_{22}^{\mathrm{T}} / \varepsilon_{0}$ & $s_{44}^{\mathrm{E}}\left(\mathrm{pm}^{2} \mathrm{~N}^{-1}\right)$ & $d_{24}\left(\mathrm{pC} \mathrm{N}^{-1}\right)$ & $p\left(10^{-4} \mathrm{C} \mathrm{m}^{-2} \mathrm{~K}^{-1}\right)$ \\
\hline Single domain rhombohedral crystal & $5000-8000$ & $200-250$ & $3000-5000$ & $5000-8000$ & $200-250$ & $3000-5000$ & $10-13$ \\
\hline Single domain orthorhombic crystal & $5000-7000$ & $200-320$ & $3500-4500$ & $12000-18000$ & $45-60$ & $1800-2200$ & - \\
\hline
\end{tabular}

piezoelectric response of single domain crystals can be used in the condition of high preload stress. Preload stress may induce phase transition and depoling in domain engineered crystals, ${ }^{22-25}$ while the thickness shear mode of single domain crystals was found to be stabilized upon the preload stress. ${ }^{26}$ Of particular significance is that the shear piezoelectric coefficient $d_{24}$ of single domain orthorhombic crystals was found to maintain similar values at a temperature of $-50{ }^{\circ} \mathrm{C}-100{ }^{\circ} \mathrm{C}$ (the $T_{\mathrm{OT}}$ phase transition temperature), with the variation being around $6 \%,{ }^{27}$ while the longitudinal piezoelectric coefficient $d_{33}$ of the [001] poled rhombohedral crystals generally exhibits $200-300 \%$ variation in the same temperature range. ${ }^{2}$ These advantages make single domain relaxor-PT crystals good candidates for various shear mode applications, such as vector sensors, non-destructive evaluation (NDE) transducers and low frequency acoustic transducers. Nevertheless, single domain crystals are prone to cracking from the electric-field-induced strain/stress during the poling process. Thus, it is desirable to investigate the poling procedure and understand the underlying cracking mechanism.

To obtain domain engineered configurations, the ferroelectric crystals are poled along specific non-polar directions, so various domains appear in the crystals. These domains can form various non- $180^{\circ}$ domain configurations, satisfying the mechanical compatibility to minimize the strain energy. ${ }^{28,29}$ For single domain crystals, however, the strain energy cannot be released by the non $-180^{\circ}$ domains. With the poling electric field applied along a spontaneous polar direction (to achieve a single domain state), the crystals will release the strain energy in an alternative way, i.e., cracking. Therefore, practically it is hard to get a crack-free single domain relaxor-PT ferroelectric crystal, which hinders the investigation and application of single domain crystals. In this paper, we compared the strainelectric field behaviors of domain engineered and single domain $0.25 \mathrm{~Pb}\left(\mathrm{In}_{0.5} \mathrm{Nb}_{0.5}\right) \mathrm{O}_{3}-0.37 \mathrm{~Pb}\left(\mathrm{Mg}_{1 / 3} \mathrm{Nb}_{2 / 3}\right) \mathrm{O}_{3}-0.38 \mathrm{PbTiO}_{3}$ (PIN-PMN-PT) tetragonal crystals and analyzed the cracking phenomenon during the poling procedure. Then, we proposed a high temperature poling technique to get crack-free single domain crystals. Finally, we compared the electromechanical properties of room temperature poled and high temperature poled crystals.

\section{Sample preparation and measurements}

The PIN-PMN-PT crystals were grown using a modified Bridgman technique at $\mathrm{XJTU}^{30}$ The composition of the crystals was determined using an electron probe X-ray microanalyzer (EPMA). The Curie temperature and coercive field were found to be $215{ }^{\circ} \mathrm{C}$ and $10 \mathrm{kV} \mathrm{cm}{ }^{-1}$, respectively. The lattice parameter $c / a$ ratio was measured by X-ray diffraction (XRD RIGAKU D/MAX-2400), being on the order of 1.015. The crystals were oriented by an RO-XRD method ${ }^{31,32}$ and cut to obtain the samples with various dimensions (thickness-mode plates, longitudinal rods, and thickness shear-mode plates) for measuring the electromechanical properties following the IEEE standard. ${ }^{33}$ Vacuum sputtered gold was applied to the polished surface as the electrodes for all the samples. The impedancefrequency behaviors were determined using an HP4294 impedance analyzer. Strain-electric field behaviors were determined using a linear variable differential transducer (LVDT) driven by a lock-in amplifier (Stanford Research System, Mode SR830). A polarizing light microscope (PLM) with a $0^{\circ} / 90^{\circ}$ crossed polarizer/analyzer (P/A) pair (Olympus BX51) was used to observe the extinction behavior of the poled samples.

\section{Poling method}

To facilitate the domain switching process, ferroelectric ceramics, such as PZT ceramics, are poled at an elevated temperature, which is higher than room temperature but much lower than the Curie temperature, being about 100-150 ${ }^{\circ} \mathrm{C}$. On the contrary, the relaxor-PT crystals are generally poled at room temperature, because the coercive fields $E_{\mathrm{c}}$ of these crystals are very low, being $2-5 \mathrm{kV} \mathrm{cm}$ (the $E_{\mathrm{c}}$ of PZT ceramics is in the range of $10-40 \mathrm{kV} \mathrm{cm}^{-1}$ ). In practice, to obtain a domain engineered structure, relaxor-PT crystals are poled at room temperature by an electric field of two-fold $E_{\mathrm{c}}$ along their nonpolar directions. However, the crystals are prone to cracking and lots of domain walls still exist when poled along a polar direction at room temperature.

In order to design a poling method for obtaining crackfree single domain crystals, the domain evolution of tetragonal crystals are analyzed by poling along the [001] and [111] directions, respectively. For tetragonal relaxor-PT crystals, six different domains, i.e., along the [001], [010], [100], [100], [010], and $[00 \overline{1}]$ directions, coexist prior to the poling process. As the crystal is poled along the [111] direction, domains along the [100], [010], and [001] directions transform to the other three electrically favored domains, forming domain engineered configuration "3T", as shown in Fig. 1(a). During the poling process, the electric-field-induced strain by domain switching is minimal, because the spontaneous strain of these six domains are equivalent with respect to the [111] direction. On 

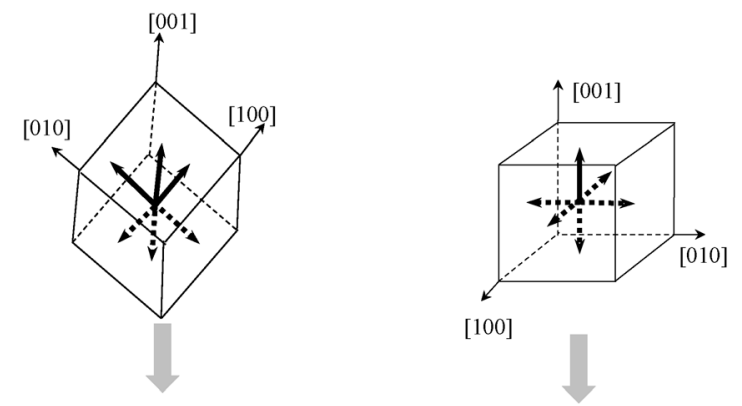

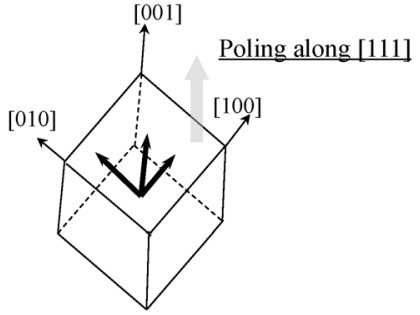

(a)

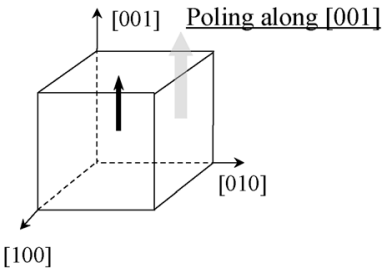

(b)
Fig. 1 Domain evolution of tetragonal crystals during the poling process. (a) Poled along the [111] direction for "3T" domain engineered configuration; (b) poled along the [001] direction to obtain a single domain crystal. The thick-black arrows represent the possible domain directions.

the contrary, as the crystal is poled along the polar [001] direction, the domains along the [010], [100], [100], [010], and [001] directions transform to the [001] domain, as shown in Fig. 1(b). Under this condition, the electric-field-induced strain is significantly large and abrupt, due to the ferroelastic $90^{\circ}$ domain switching from [010], [100], [100] or [010] domain to [001] domain.

Fig. 2 shows the strain-electric field behaviors for [001] and [111] oriented tetragonal PIN-PMN-PT crystals. When the applied field is around $E_{\mathrm{c}}$, six possible domains exist in the crystal, which can be regarded as the crystal state prior to poling. In the high electric field region, only the domains with the direction closest to poling direction exist, which is the

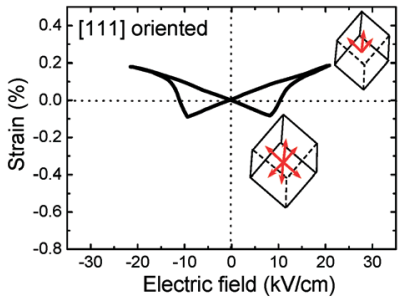

(a)

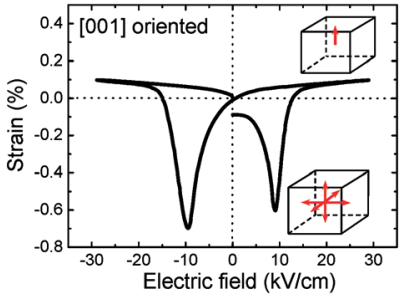

(b)
Fig. 2 The strain-electric field behaviors of (a) [111] and (b) [001] oriented tetragonal $0.25 \mathrm{PIN}-0.37 \mathrm{PMN}-0.38 \mathrm{PT}$ crystals, where the possible domains at different electric fields are shown by the red arrows. At coercive field, six possible domains appear in the crystal, which can be regarded as the crystal state prior to poling. In the high electric field region, only the domains closest to poling direction exist, which is the state of the crystal after poling. crystal state after poling. As expected, for [001] oriented crystals the negative strain is found to be $0.7 \%$, with a sharp strain-electric field behavior at $E_{\mathrm{c}}$, being attributed to the $90^{\circ}$ domain switching, while the negative strain of [111] oriented crystals was only $0.08 \%$, which is mainly contributed by the linear piezoelectric effect, being equal to $-d_{33} E_{\mathrm{c}}$. It is interesting to note that if all the domains are along the [010], [010], [100], or [1̄00] direction before poling, the contribution of the $90^{\circ}$ domain switching to the strain should be equal to the ratio of $(c-a) / a=1.5 \%$ ( $c$ and $a$ are the lattice parameters). However, the reality is that before poling some domains are along the [001] and [001] directions, which can explain that the contribution of the $90^{\circ}$ domain switching to the strain is lower than the ratio of $(c-a) / a$ in the [001] oriented tetragonal crystal.

According to the above discussion, the large strain variation is thought to be the dominant factor for the cracking phenomenon when the crystals transform into a single domain state. The large strain is induced by the ferroelastic domain switching (i.e., non- $180^{\circ}$ ferroelectric domain switching), which should be avoided during the poling process, in order to reduce the cracking. Based on this idea, a high temperature poling approach is proposed to obtain single domain crystals. As described in Fig. 3, first the crystal was heated above the Curie temperature (from room temperature to $250{ }^{\circ} \mathrm{C}$ at the rate of $5{ }^{\circ} \mathrm{C} \mathrm{min}^{-1}$ ). Then, an electric field of $2-3 \mathrm{kV} \mathrm{cm}^{-1}$ was applied to the [001] oriented tetragonal crystal. Finally, the crystal was slowly cooled down to room temperature with the applied dc electric field at the rate of $2{ }^{\circ} \mathrm{C} \mathrm{min}^{-1}$. During this poling process, only the domain along the [001] direction is expected to form through the paraelectric-ferroelectric phase transition point $\left(T_{\mathrm{C}}\right)$. Meanwhile, in this process, the rate of strain change can be controlled by the temperature decreasing rate. Compared with the room temperature poling process, therefore, $90^{\circ}$ domain switching and abrupt strain variation are precluded in the above poling process.

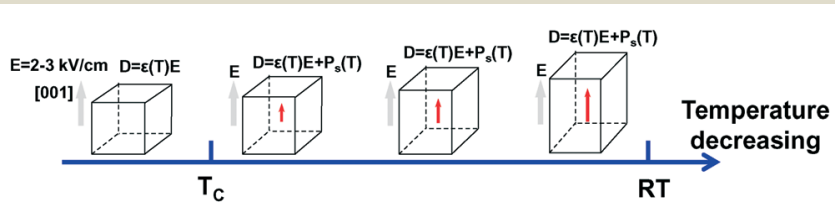

Fig. 3 Schematic diagram of the high temperature poling technique. The electric displacement is expressed at various temperatures. The red arrows represent the spontaneous polarization for the tetragonal crystal. At a temperature higher than $T_{C}$, the crystal is in the paraelectric phase, where the spontaneous polarization and ferroelectric domains cannot be induced by an electric field and the electric displacement only comes from a linear dielectric response, being equal to $\varepsilon(T) E$. As the temperature decreases below $T_{C}$, the spontaneous polarization along the direction of the electric field ([001] direction) appears, and the electric displacement can be expressed as $\varepsilon(T) E+P_{\mathrm{S}}(T)$. With further decrease in temperature, the spontaneous polarization and strain increase gradually. 


\section{Comparison of tetragonal PIN-PMN-PT single domain crystals poled by a general method (room temperature poling) and a high temperature poling method}

Fig. 4(a1) and (b1) show the images of tetragonal PIN-PMN-PT crystals poled by room-temperature and high-temperature techniques, respectively. A high density of domain walls and cracks was observed in the room-temperature poled crystals, while the high-temperature poled crystals do not show any cracks. It should be noted that some domain walls are still present around the surface of the high-temperature poled crystals. These domain walls are hard to be removed, because the applied electric field near the surface deviates a lot from the ideal situation, i.e., the direction of the applied electric field is not strictly parallel to the [001] direction on the (a1)
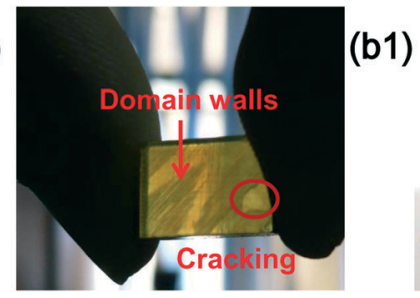

(a2)

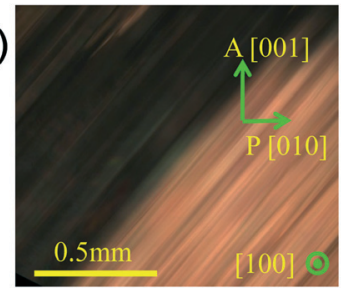

(a)

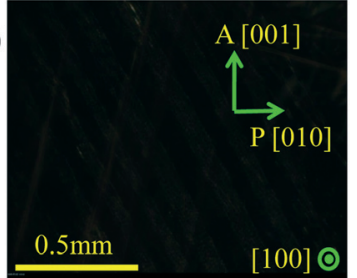

(b)

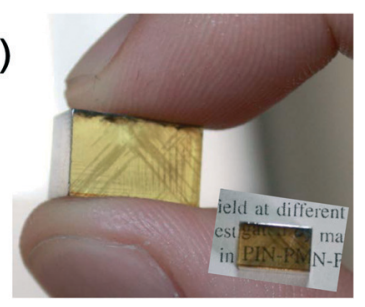

Fig. 4 Images of 0.25PIN-0.37PMN-0.38PT crystals poled by (a) room temperature and (b) high temperature techniques, respectively. (a1) and (b1) were taken with a camera. (a2) and (b2) were taken using a polarizing light microscope with a $0 \% / 90^{\circ}$ crossed polarizer/analyzer (P/A) pair. In these pictures, the dimension of the samples is $6 \mathrm{~mm}$ $([100]) \times 12 \mathrm{~mm}([010]) \times 8 \mathrm{~mm}([001])$. The samples are poled along the polar [001] direction, and the (100) surfaces are optically polished for domain observation. proximity of the sample surface. Fig. 4(a2) and (b2) show the PLM images of the [001] poled tetragonal PIN-PMN-PT, where the light propagates along the [100] direction, and the polarizer and analyser are set along the [010] and [001] directions, respectively. In principle, six possible domains of the tetragonal crystal should exhibit extinction as the polarized-light propagates along the [100] direction with the polarizer/analyzer being along the $[010] /[001]$ directions. ${ }^{34}$ However, due to the influence of the high density of domain walls (the symmetry of the domain wall is lower than that of the tetragonal domain ${ }^{35,36}$ ), a room-temperature poled crystal does not show an extinction phenomenon, again indicating that the density of the domain walls in room-temperature poled crystals is much higher than that in the high-temperature poled counterpart.

Table 2 shows the electromechanical properties of the longitudinal mode tetragonal PIN-PMN-PT crystals. Although the dielectric permittivity, piezoelectric coefficient and coupling factor are similar for room temperature and high temperature poled crystals, the dielectric and mechanical losses, which are mainly attributed to the domain wall motion, ${ }^{37-40}$ are much higher for the room temperature poled crystals.

The effects of incomplete poling and domain walls are more obvious in the strain-electric field and impedance-frequency behaviors for the tetragonal PIN-PMN-PT crystals. As shown in Fig. 5(a1) and (b1), a nearly linear strain-electric field response was observed for high temperature poled crystals, while a large strain hysteresis $(\sim 40 \%)$ induced by the domain wall motion was shown in the room temperature poled counterpart. Fig. 5(a2) and (b2) show the impedance and phase spectra for the thickness shear vibration mode (15-mode). The clean shear-mode resonance and antiresonance peaks were observed in the high temperature poled crystal. For the room temperature poled crystal, however, some other vibrations (i.e., thickness vibration) might be coupled with the thickness shear-mode, because the domains along the [100] or [010] direction yet exist. Meanwhile, the homogeneity of the electromechanical properties may be affected by uneven distribution of domains and domain walls in the room temperature poled crystal, as depicted in Fig. 4(a1). Due to the above two factors, noisy impedance-peaks were observed in the room temperature poled samples. The large strain hysteresis and noisy peaks of the room temperature poled piezoelectric crystals can greatly restrict their electromechanical applications.

Table 2 Longitudinal electromechanical properties of [001] poled tetragonal 0.25PIN-0.37PMN-0.38PT crystals. Plate samples with dimensions of $10 \mathrm{~mm} \times 10 \mathrm{~mm} \times 1 \mathrm{~mm}$ (poling direction) were used for measuring the dielectric permittivity and losses. Longitudinal rods with dimensions of $8 \mathrm{~mm}$ (poling direction) $\times 2 \mathrm{~mm} \times 2 \mathrm{~mm}$ were used for measuring the $d_{33}, k_{33}$ and mechanical quality factor. Mechanical quality factor $Q_{\mathrm{m}}$ is the inverse of the mechanical loss

\begin{tabular}{|c|c|c|c|c|c|}
\hline Materials & $\begin{array}{l}\text { Dielectric permittivity } \\
\varepsilon_{33}^{\mathrm{T}} / \varepsilon_{0}\end{array}$ & $\begin{array}{l}\text { Piezoelectric coefficient } \\
d_{33}\left(\mathrm{pC} \mathrm{N}^{-1}\right)\end{array}$ & $\begin{array}{l}\text { Coupling factor } \\
k_{33}\end{array}$ & $\begin{array}{l}\text { Dielectric loss } \\
\tan \delta\end{array}$ & $\begin{array}{l}\text { Mechanical quality } \\
\text { factor } Q_{\mathrm{m}}\end{array}$ \\
\hline Room temperature poled crystal & $1100-1400$ & $450-480$ & $83 \%$ & $0.5-2 \%$ & $300-700$ \\
\hline High temperature poled crystal & $1000-1200$ & $450-470$ & $84 \%$ & $0.1-0.3 \%$ & $1500-2500$ \\
\hline
\end{tabular}



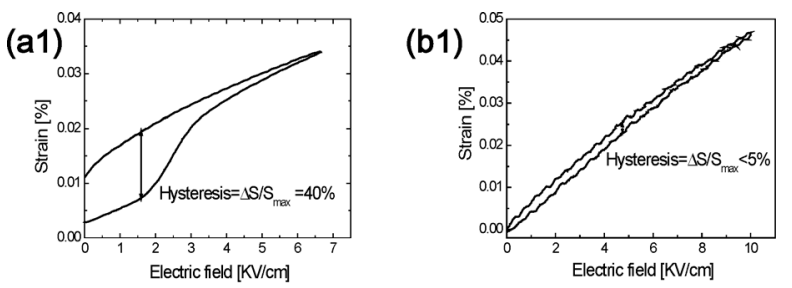

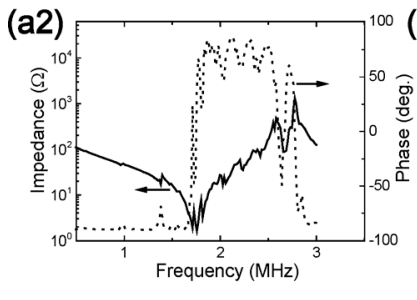

(a)

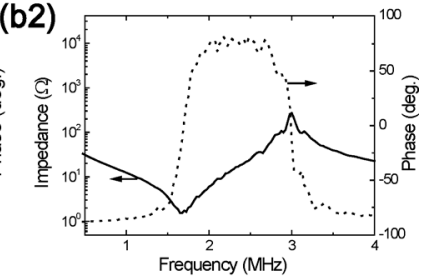

(b)
Fig. 5 Electromechanical properties of [001] oriented $0.25 \mathrm{PIN}-0.37 \mathrm{PMN}-0.38 \mathrm{PT}$ crystals poled by (a) room-temperature and (b) high-temperature techniques, respectively. (a1) and (b1) are the unipolar strain-electric field response. (a2) and (b2) are the impedance and phase spectrum of the thickness shear mode. The longitudinal rods with dimensions of $5 \mathrm{~mm}$ (poling direction) $\times 1.5 \mathrm{~mm} \times 1.5 \mathrm{~mm}$ are used for strain-electric field measurements. The shear thickness plates with dimensions of $5 \mathrm{~mm}$ (poling direction) $\times 5 \mathrm{~mm} \times 0.5 \mathrm{~mm}$ are used for measuring the impedance and phase spectrum of the 15 -mode. The shear piezoelectric coefficients $d_{15}$ of the room-temperature and high-temperature poled crystals are 1800 and $2000 \mathrm{pC} \mathrm{N}^{-1}$, respectively.

\section{Conclusions}

The [001] and [111] oriented 0.25PIN-0.37PMN-0.38PT tetragonal crystals poled using different conditions were investigated; the underlying cracking mechanism in single domain crystals was attributed to the large strain variation induced by ferroelastic domain switching. A feasible high temperature poling technique was proposed, by which a quasi-single domain tetragonal PIN-PMN-PT crystal was obtained without cracks. Compared to the room temperature poled crystals, high temperature poled crystals exhibited a low density of domain walls, leading to a greatly reduced dielectric loss, strain hysteresis and an increased mechanical quality factor, without sacrificing the piezoelectric properties. The proposed method may also benefit other perovskite ferroelectric crystals for achieving a single domain state, such as barium titanate and potassium-sodium niobate crystals.

\section{Acknowledgements}

The authors want to thank Professor Thomas R. Shrout for his valuable suggestions. This work was supported by the National Nature Science Foundation of China (grant no. 51002116, 51102193, 51202183 and 51372196), the China Postdoctoral Science Foundation and the Fundamental Research Funds for the Central Universities.

\section{References}

1 S. E. Park and T. R. Shrout, J. Appl. Phys., 1997, 82, 1804.

2 S. Zhang and F. Li, J. Appl. Phys., 2012, 111, 031301.
3 X. Li and H. Luo, J. Am. Ceram. Soc., 2010, 93, 2915.

4 N. Luo, Y. Li, Z. Xia and Q. Li, CrystEngComm, 2012, 14, 4547.

5 F. Li, S. Zhang, Z. Xu, X. Wei and T. R. Shrout, J. Appl. Phys., 2011, 109, 014108.

6 G. S. Xu, K. Chen, D. F. Yang and J. B. Li, Appl. Phys. Lett., 2007, 90, 032901.

7 D. Damjanovic, IEEE Trans. Ultrason. Ferroelectr. Freq. Control, 2009, 56, 1574.

8 F. Li, S. Zhang, Z. Xu, X. Wei, J. Luo and T. R. Shrout, J. Appl. Phys., 2010, 108, 034106.

9 M. Davis, D. Damjanovic, D. Hayem and N. Setter, J. Appl. Phys., 2005, 98, 014102.

10 X. Jiang, P. W. Rehrig, W. S. Hackenberger, E. Smith, S. Dong, D. Viehland, J. Moore and B. Patrick, Proc. SPIE Smart Struct. Mater., 2005, 5761, 253.

11 K. Kim, S. Zhang and X. Jiang, Appl. Phys. Lett., 2012, 100, 253501.

12 X. Jiang, P. W. Rehrig, W. S. Hackenberger and T. R. Shrout, Adv. Cryog. Eng., 2006, 51A\&B, 1783.

13 D. Damjanovic, J. Am. Ceram. Soc., 2005, 88, 2663.

14 R. Zhang, B. Jiang, W. Cao and A. Amin, J. Mater. Sci. Lett., 2002, 21, 1877.

15 F. Li, S. Zhang, Z. Xu, X. Wei and T. R. Shrout, Adv. Funct. Mater., 2011, 21, 2118.

16 S. Zhang, F. Li, J. Luo, R. Sahul and T. R. Shrout, IEEE Trans. Ultrason. Ferroelectr. Freq. Control, 2013, 60, 1572.

17 S. F. Liu, W. Ren, B. K. Mukherjee, S. J. Zhang, T. R. Shrout, P. W. Rehrig and W. S. Hackenberger, Appl. Phys. Lett., 2003, 83, 2886.

18 J. Jin, K. K. Rajan and L. Lim, IEEE Trans. Ultrason. Ferroelectr. Freq. Control, 2007, 54, 2222.

19 M. Davis, D. Damjanovic and N. Setter, J. Appl. Phys., 2004, 96, 2811.

20 Y. X. Tang, X. Y. Zhao, X. Q. Feng, W. Q. Jin and H. S. Luo, Appl. Phys. Lett., 2005, 86, 082901.

21 L. Luo, H. Wang, Y. Tang, X. Zhao, Z. Feng, D. Lin and H. S. Luo, J. Appl. Phys., 2006, 99, 024104.

22 J. Gao, Z. Xu, F. Li, C. Zhang, Z. Li, X. Wu, L. Wang, Y. Liu, G. Liu and H. He, J. Appl. Phys., 2011, 110, 106101.

23 A. Amin, IEEE Trans. Ultrason. Ferroelectr. Freq. Control, 2005, 52, 1632.

24 Q. Wan, C. Chen and Y. P. Shen, J. Appl. Phys., 2005, 98, 024103.

25 D. Viehland, L. Ewart, J. Powers and J. F. Li, J. Appl. Phys., 2001, 90, 2479.

26 F. Li, S. Zhang, Z. Xu, D. Lin, J. Gao, Z. Li and L. Wang, Appl. Phys. Lett., 2012, 100, 192901.

27 F. Li, S. Zhang, Z. Xu, X. Wei, J. Luo and T. R. Shrout, Appl. Phys. Lett., 2010, 97, 252903.

28 R. W. Cahn, Adv. Phys., 1954, 3, 202.

29 J. Fousek and V. Janovec, J. Appl. Phys., 1969, 40, 135.

30 X. Wang, Z. Xu, Z. Li and F. Li, Ferroelectrics, 2010, 401, 173.

31 F. Li, L. Jin, Z. Xu and Z. Guo, Rev. Sci. Instrum., 2009, 80, 085106.

32 Z. Guo, L. Jin, F. Li and Y. Bai, J. Phys. D: Appl. Phys., 2009, 42, 012001. 
33 IEEE Standard on Piezoelectricity, "IEEE Standard on Piezoelectricity", ANSI/IEEE Std 176, 1987.

34 R. R. Chien, V. H. Schmidt, C. Tu, L. W. Hung and H. Luo, Phys. Rev. B: Condens. Matter Mater. Phys., 2004, 69, 172101.

35 C. Jia, K. W. Urban, M. Alexe, D. Hesse and I. Vrejoiu, Science, 2011, 331, 1420.

36 P. V. Yudin, A. K. Tagantsev and N. Setter, Phys. Rev. B: Condens. Matter Mater. Phys., 2013, 88, 024102.
37 K. Uchino, J. Zheng, Y. Chen, X. Du, J. Ryu, Y. Gao, S. Ural, S. Priya and S. Hirose, J. Mater. Sci., 2006, 41, 217.

38 K. Uchino and S. Hirose, IEEE Trans. Ultrason. Ferroelectr. Freq. Control, 2001, 48, 307.

39 D. Damjanovic and M. Demartin, J. Phys.: Condens. Matter, 1997, 9, 4943.

40 L. Jin, V. Porokhonskyy and D. Damjanovic, Appl. Phys. Lett., 2010, 96, 242902. 